\title{
LA NUEVA DIRECTIVA EUROPEA SOBRE SEGURIDAD Y SALUD EN LAS OBRAS Y EL MODELO ESPAÑOL
}

\author{
(THE NEW EUROPEAN DIRECTIVE ON SAFETY AND HEALTH IN WORKS AND \\ THE SPANISH MODEL)
}

Rafael Anduiza Arriola*, Arquitecto Técnico.

Profesor Titular de la Universidad Politécnica de Madrid ESPAÑA

Fecha de recepción: 2-IX-92

RESUMEN
Análisis crítico de la Normativa de la Comunidad Económica Europea
sobre seguridad y salud laboral, en obras de construcción, en
comparación con la Normativa española.
SUMMARY

Critical analysis of the The European Economic Community Ordinance on Labour Safety and Health in Construction works in comparison with the Spanish Ordinance.
El Consejo de las Comunidades Europeas adoptó, el 24 de junio de 1992, la "Directiva 92/57/CEE relativa a las disposiciones mínimas de seguridad y salud que deben aplicarse en obras de construcción temporales o móviles" (en lo sucesivo Directiva de Obras).

Los estados miembros deberán poner en vigor las disposiciones legales y administrativas para dar cumplimiento a lo dispuesto en la Directiva de Obras, a más tardar el 31 de diciembre de 1993 (Artículo 14.1).

Esta nueva Directiva de Obras tiene su origen en el apartado 1, del artículo 16 de la Directiva Marco 89/391/CEE, relativa a la aplicación de medidas para promover la mejora de la seguridad y salud de los trabajadores en el trabajo, en el que se establece la adopción por el Consejo, a propuesta de la Comisión, de Directivas específicas en diversos ámbitos y, entre ellas, el de las obras temporales y móviles.

*Director del Proyecto "Caupolicán" de "Investigación en seguridad y salud en construcción".
De entre los 15 considerandos que se formulan, destacamos los siguientes:

- Considerando que las obras de construcción, temporales o móviles, constituyen un sector de actividad que implica riesgos particularmente elevados para los trabajadores.

- Considerando que más de la mitad de los accidentes de trabajo, en las obras de construcción en la Comunidad, está relacionada con decisiones arquitectónicas y/o de organización inadecuadas o con una mala planificación de las obras en su fase de proyecto.

- Considerando que en todos los Estados miembros se debe informar, antes del inicio de los trabajos, a las autoridades competentes en materia de seguridad y de salud en el trabajo acerca de la realización de obras cuya importancia supere un determinado umbral.

- Considerando que, durante la ejecución de un proyecto, la falta de coordinación debida en particular a la participación simultánea o sucesiva de empresas diferentes en una misma obra de construcción temporal o móvil, puede dar lugar a un número elevado de accidentes de trabajo. 
- Considerando, por ello, que resulta necesario reforzar la coordinación entre las distintas partes que intervienen ya desde la fase de proyecto, pero igualmente durante la ejecución de la obra.

- Considerando, por otra parte, que los trabajadores autónomos y los empresarios, cuando ellos mismos ejercen una actividad profesional en una obra de construcción temporal o móvil, pueden poner en peligro, por dichas actividades, la seguridad y la salud de los trabajadores.

Que claramente hacen referencia a los siguientes aspectos:

- Comunicar a las autoridades competentes, antes del inicio de las obras, la actividad productiva.

- Inserción de la seguridad en el Proyecto.

- Reforzar la coordinación entre las diversas empresas en la ejecución de la obra y entre los agentes relacionados con el proyecto.

- Tratamiento específico y explícito de los trabajadores independientes.

Esta Directiva circunscribe el ámbito de aplicación a las obras en las que se efectúen trabajos de construcción. o ingeniería que se relacionan de forma no exhaustiva en el Anexo I, que dice:

\section{ANEXO I}

RELACIÓN NO EXHAUSTIVA DE LAS OBRAS DE CONSTRUCCIÓN O DE INGENIERÍA CIVIL (MENCIONADA EN LA LETRA A) DEL ARTÍCULO 2.

1. Excavación.

2. Movimiento de Tierras.

3. Construcción.

4. Montaje y desmontaje de elementos prefabricados.

5. Acondicionamiento o instalaciones.

6. Transformación.

7. Rehabilitación.

8. Reparación.

9. Desmantelamiento.

10. Derribo.

11. Mantenimiento.
12. Conservación - Trabajos de pintura y de limpieza. 13. Saneamiento.

Relativamente novedosas resultan las figuras de los coordinadores de seguridad y salud en la elaboración del proyecto y durante la realización de la obra construida, definidas en el Artículo 2:

e) Coordinador en materia de seguridad y de salud durante la elaboración del proyecto de construcción, cualquier persona física o jurídica designada por la propiedad y/o por el director de la obra para llevar a cabo, durante la fase de proyecto de la obra, las tareas que se mencionan en el artículo 5.

f) Coordinador en materia de seguridad y de salud durante la realización de la obra, cualquier persona física o jurídica designada por la propiedad y/o por el director de la obra para llevar a cabo, durante la realización de la obra, las tareas que se mencionan en el artículo 6.

La propiedad o el director de la obra (entiéndase "persona física o jurídica encargada del proyecto y/o de la ejecución y/o del control de la ejecución de la obra por cuenta de la propiedad") nombrará uno o varios coordinadores, en el caso de obras en las que estén presentes varias empresas.

La propiedad o el director de obra cursará un aviso previo a las autoridades competentes antes del comienzo de los trabajos, en los siguientes casos:

- Cuando la duración sea superior a 30 días laborables y empleen a más de 20 trabajadores simultáneamente.

- Cuando el volumen estimado sea superior a 500 hombres/día.

Este aviso estará expuesto en la obra y se actualizará en caso necesario. Su contenido se explicita en el correspondiente anexo, que dice:

ANEXO III

CONTENIDO DEL AVISO PREVIO MENCIONADO EN EL PÁRRAFO PRIMERO DEL APARTADO 3 DEL ARTICULO 3.

1. Fecha de comunicación. 
2. Dirección exacta de la obra.

3. Propiedad (nombre/s) y dirección/es).

4. Tipo de obra.

5. Director/es de la obra (nombre/s y dirección/es).

6. Coordinador/es en materia de seguridad y de salud durante la elaboración del proyecto de la obra (nombre/s y dirección/es).

7. Coordinador/es en materia de seguridad y salud durante la ejecución de la obra (nombre/s y dirección/es).

8. Fecha prevista para el comienzo de los trabajos en la obra.

9. Duración prevista de los trabajos en la obra.

10. Número máximo estimado de trabajadores en la obra.

11. Número previsto de empresas y trabajadores autónomos en la obra.

12. Datos de identificación de las empresas ya seleccionadas.

Antes del comienzo de las obras, la propiedad o el director de la obra velará para que se establezca un plan de seguridad y salud en el que precisen las normas aplicables en dicha obra, considerando cualquier actividad que se lleve a cabo in situ, conteniendo además medidas específicas relativas a los siguientes trabajos.

\section{ANEXO II}

RELACIÓN NO EXHAUSTIVA DE LOS TRABAJOS QUE IMPLICAN RIESGOS ESPECÍFICOS PARA LA SEGURIDAD Y LA SALUD DE LOS TRABAJADORES (MENCIONADA EN EL PÁRRAFO SEGUNDO DEL APARTADO 2 DEL ARTÍCULO 3)

1. Trabajos que expongan a los trabajadores a riesgos de todo tipo de sepultamiento, de hundimiento o de caída de altura, particularmente agravados por la naturaleza de las actividades o de los procedimientos aplicados, o por el entorno del puesto de trabajo o de la obra.

2. Trabajos que expongan a los trabajadores a sustancias químicas o biológicas que presenten especial riesgo para la seguridad y la salud de los trabajadores o que requieran una exigencia legal de vigilancia de la salud.

3. Trabajos con radiaciones ionizantes que exijan la designación de zonas controladas o vigiladas, tal como se define en el artículo 20 de la Directiva del Consejo 80/836/Euratom.

4. Trabajos en la proximidad de líneas eléctricas de alta tensión.

5. Trabajos que expongan a riesgo de ahogamiento por inmersión.

6. Obras de excavación de pozos, de movimientos de tierras subterráneos y de túneles.

7. Trabajos realizados en inmersión con equipo subacuático.

8. Trabajos reálizados en cajones de aire comprimido.

9. Trabajos que impliquen el uso de explosivos.

10. Trabajos que requieran montar o desmontar elementos prefabricados pesados.

Además de la Notificación y el Plan de Seguridad y Salud, se prevé la constitución de un Expediente adaptado a las características de la obra, en el que se indiquen los elementos útiles en materia de seguridad y salud que deberán tomarse en consideración, en caso de realización de trabajos posteriores.

La transposición de la Directiva deberá precisar con mayor exactitud las condiciones de obligatoriedad para la existencia del plan, si bien, en principio, hay que entender que se extiende a las obras que precisan de notificación y/o las que se realicen de trabajos señalados en el Anexo II, anteriormente citado.

Los principios generales de seguridad y salud a tener en cuenta por el autor del proyecto (o la propiedad, en su caso) en la "concepción, estudio y elaboración del proyecto de la obra", son los que se contienen en la Directiva Marco, y en particular:

- Al tomar las decisiones arquitectónicas, técnicas y/o de organización, con el fin de planificar los distintos trabajos o fases de trabajo que se desarrollarán simultánea o sucesivamente.

- Al estimar la duración requerida para la ejecución de estos distintos trabajos o fases de trabajo. 
Tanto el Plan como el Expediente deberán adaptarse en función de la evolución de los trabajos y las modificaciones que se produzcan.

En la elaboración del proyecto, los coordinadores de Seguridad en el proyecto, tienen asignadas las siguientes tareas:

a) Coordinar la aplicación en los principios generales y específicos en materia de seguridad y salud.

b) Establecer o hacer que se establezca el plan de Seguridad.

c) Constituir el expediente.

Durante la ejecución de la obra, los coordinadores de esta fase realizarán las siguientes tareas:

A) Coordinarán la aplicación de los principios generales de prevención y de seguridad:

- Al tomar las decisiones técnicas y/o de organización con el fin de planificar los distintos trabajos o fases de trabajo que vayan a desarrollarse simultánea o sucesivamente.

- $\mathrm{Al}$ estimar la duración requerida para la ejecución de estos distintos trabajos o fases de trabajo.

B) Coordinarán la aplicación de las disposiciones pertinentes, con el fin de garantizar que los empresarios $\mathrm{y}$, si ello fuera necesario, para la protección de los trabajadores, los trabajadores autónomos:

- apliquen de manera coherente los principios que se mencionan en el artículo 8 (que son los contenidos en el artículo 6 de la Directiva marco y de los que resaltamos: detección, evaluación y supresión de riesgos. Prioridad de aplicación de medidas correctoras de carácter colectivo. Instrucción e información a los trabajadores, considerando sus capacidades y sin cargo económico alguno para ellos), y con carácter específico se aplicarán en lo que respecta:

a) al mantenimiento de la obra en buen orden y en estado satisfactorio de salubridad,

b) a la elección del emplazamiento de los puestos de trabajo, teniendo en cuenta sus condiciones de acceso, y la determinación de las vías o zonas de desplazamiento o circulación,

c) a las condiciones de manipulación de los distintos materiales,

d) al mantenimiento, al control antes de la puesta en servicio y al control periódico de las instalaciones y dispositivos, con objeto de suprimir los defectos que pudieran afectar a la seguridad y a la salud de los trabajadores,

e) a la delimitación y al acondicionamiento de las zonas de almacenamiento y depósito de los distintos materiales, en particular si se trata de materias o sustancias peligrosas,

f) a las condiciones de recogida de los materiales peligrosos que se hayan utilizado,

g) al almacenamiento y a la eliminación o evacuación de los residuos y de los escombros,

h) a la adaptación, en función de la evolución de la obra, del período de tiempo efectivo que habrá de dedicarse a los distintos tipos de trabajos o fases de trabajo,

i) a la cooperación entre los empresarios y los trabajadores autónomos,

j) a las interacciones con cualquier otro tipo de actividad que se realice in situ o cerca del lugar de la obra;

- apliquen, cuando se requiera, el plan de seguridad y de salud considerado en la letra b) del artículo 5 .

C) Procederán o harán que se proceda a las posibles adaptaciones del plan de seguridad y de salud que se menciona en la letra b) del artículo 5 y del expediente contemplado en la letra c) del artículo 5, en función de la evolución de los trabajos y de las modificaciones que pudieran haberse producido.

D) Organizarán entre los empresarios, incluidos los que intervengan en la obra, la cooperación y coordinación de las actividades con vistas a la protección de los trabajadores y a la prevención de accidentes y riesgos profesionales que puedan atentar contra la sa- 
lud, así como su información mutua, previstas en el apartado 4 del artículo 6 de la Directiva 89/391/CEE incluyendo, en su caso, a los trabajadores autónomos.

E) Coordinarán el control de la aplicación correcta de los métodos de trabajo.

F) Adoptarán las medidas necesarias para que sólo las personas autorizadas puedan acceder a la obra.

Las obligaciones que la Directiva asigna a los empresarios se concretan en la aplicación de lo contenido en el artículo 8 que hemos sintetizado anteriormente y lo contenido en el anexo IV, que más adelante comentaremos.

Asimismo, están obligados a tener en cuenta las indicaciones del coordinador o coordinadores.

Llama la atención el intenso tratamiento que se da en el artículo 10 a los trabajadores autónomos y a los empresarios que ejercen ellos mismos una actividad profesional. En ambos casos se tendrán en cuenta las indicaciones de los coordinadores y se exige sistemática y pormenorizadamente la aplicación de lo contenido en diversos artículos de la Directiva Marco 89/391/CEE y de las Directivas 89/655/CE y 89/656/CEE, relativas a la utilización de equipos de trabajo y de equipos de protección individual, respectivamente.

Por lo que se refiere a la información, consulta y participación de los trabajadores, se destaca que la información deberá ser comprensible para los trabajadores, y en cuanto a la participación se tiene en cuenta el nivel de riesgo y de la importancia de la obra.

Consideramos muy interesante transcribir el artículo 7, en el que se establecen las responsabilidades de la propiedad, los directores de obra y los empresarios, que subsisten, aún cuando hayan procedido al nombramiento de los coordinadores, cuyas tareas se concretan en los artículos 5 y 6 de la Directiva.

\section{Artículo 7.-Responsabilidades de la propiedad, de los directores de obra y de los empresarios.}

1. Si la propiedad o el director de obra hubiere designado a uno o varios coordinadores para ejecutar las tareas mencionadas en los artículo 5 y 6 , ello no le eximirá de sus responsabilidades en este ámbito.

2. La aplicación de los artículos 5 y 6 del apartado 1 del presente artículo no afectará al principio de responsabilidad de los empresarios prevista en la Directiva 89/391/CEE.

El ANEXO IV se titula "Disposiciones mínimas de Seguridad y Salud que deberán aplicarse en las obras", y se divide en dos partes:

Parte A: Disposiciones mínimas generales relativas a los lugares de trabajo en las obras.

1. Estabilidad y solidez.

2. Instalaciones y reparto de energía.

3. Vías de salida y emergencia.

4. Detección y lucha contra incendios.

5. Ventilación.

6. Exposición a riesgos particulares.

7. Temperatura.

8. Iluminación natural y artificial de los lugares de trabajo, de los locales y de las vías de circulación en obra.

9. Puertas y portones.

10. Vías de circulación-zonas peligrosas.

11. Muelles y rampas de carga.

12. Espacio necesario para la libertad de movimiento en el lugar de trabajo.

13. Primeros auxilios.

14. Equipos sanitarios.

15. Locales de descanso y/o alojamiento.

16. Mujeres embarazadas y madres lactantes.

17. Trabajadores minusválidos.

18. Disposiciones varias.

Estas obligaciones se aplicarán siempre que lo exijan las características de la obra, la actividad, las circunstancias o cualquier riesgo.

Parte B: Disposiciones mínimas específicas, relativas a los puestos de trabajo en las obras.

Sección I: Puestos de trabajo en las obras en el interior de los locales.

1. Estabilidad y solidez.

2. Puertas de emergencia. 
3. Ventilación.

4. Teperatura.

5. Iluminación natural y artificial.

6. Suelos, paredes y techos de los locales.

7. Ventanas y vanos de iluminación central.

8. Puertas y portones.

9. Vías de circulación.

10. Medidas específicas para escalera y cintas rodantes.

11. Dimensiones y volumen de aire de los locales.

Sección II: Puestos de trabajo en las obras en el exterior de los locales.

1. Estabilidad y solidez.

2. Instalaciones de distribución de energía.

3. Factores atmosféricos.

4. Caídas de objetos.

5. Caídas de altura.

6. Andamios y escaleras.

7. Aparatos elevadores.

8. Vehículos y maquinaria para movimientos de tierras y para manipulación de materiales.

9. Instalaciones, máquinas y equipos.

10. Excavaciones, pozos, trabajos subterráneos, túneles, movimientos de tierras.

11. Trabajos de derribo.

12. Armaduras metálicas o de hormigón, encofrados y elementos prefabricados pesados.

13. Ataguías y cajones de aire comprimido.

14. Trabajos sobre tejados.

Esta clarificación de disposiciones mínimas divididas en las dos secciones, no deberá considerarse imperativa cuando lo requieran las situaciones particulares.

Tras este repaso de la Directiva de Obras, por cierto de lectura bastante ingrata, por la contínua referencia a otras Directivas, cabe preguntarse, de qué forma va a afectar al actual modelo español y a las disposiciones vigentes.

En primer lugar, hay que destacar que es una buena ocasiòn par reordenar la dispersa y antigua normativa. Sobre todo es necesario simplificarla y hacerla cumplible.

El Real Decreto 555/86, por el que se establece la obligación de incluir un Estudio de Seguridad en los proyectos, así como la redacción por el constructor (o por el propietario en su caso) de un Plan de Seguridad en determinado tipo de obra, va a precisar de los correspondientes retoques.
En efecto, los parámetros determinantes de la obligación de inclusión del Estudio de Seguridad y de redacción del correspondiente Plan, son:

a) Obras cuyo presupuesto global sea igual o superior a 100 millones de pesetas.

b) Concurrencia de 50 o más trabajadores en la obra.

c) Obras de túneles, galerías, conducciones subterráneas o de riesgo especial.

Por tanto, estos límites han de modificarse, ampliándose a las obras que precisan de Notificación, Plan y Expediente.

Como se ha visto, la acción del coordinador en la fase del proyecto, ha de inspirarse en los principios básicos de la Directiva Marco, que en su artículo 6, y del que a efectos de la comparación que establecemos, destacamos los siguientes enunciados como obligaciones de los empresarios:

6.2.-a) evitar riesgos,

b) evaluar los riesgos que no se pueden evitar,

c) combatir los riesgos en su origen,

e) tener en cuenta la evolución técnica,

g) planificar la prevención, buscando un conjunto coherente que integre en ella la técnica, la organización del trabajo, las relaciones sociales y los factores ambientales en el trabajo,

h) adoptar medidas que antepongan la protección colectiva a la individual;

6.3.-a) evaluar los riesgos para la seguridad y la salud de los trabajadores, en lo que se refiere a la elección de los equipos de trabajo, de las sustancias o preparados químicos, y el acondicionamiento de los lugares de trabajo.

En el artículo 8, en relación con los primeros auxilios, lucha contra incendios, evacuación de los trabajadores, riesgo grave e inminente, cita:

8.1.-...adoptar en materia de primeros auxilios, lucha contra incendios y evacuación de trabajadores las medidas necesarias, adaptadas al tamaño y al carácter de las actividades de la empresa y/o el establecimiento, habida cuenta de que otras personas pueden encontrarse presentes... 
Como se ve, todos estos principios y otros matices que se contienen en el texto de la Directiva, tienen su fiel reflejo en el estudio de Seguridad del R.D. 555, especialmente en lo que se explicita en el contenido de la Memoria y el Pliego de condiciones, que respectivamente dicen:

a) Memoria descriptiva de los procedimientos y equipos técnicos a utilizar, con relación de los riesgos de accidentes y enfermedades profesionales que, presumiblemente, pueden producirse; especificación de las medidas preventivas y protecciones técnicas tendentes a evitarlos y evaluación de su eficacia, en especial cuando se propogan medidas alternativas; asimismo se incluirá la descripción de los servicios sanitarios y comunes de que deberá estar dotado el centro de trabajo de la obra.

b) Pliego de condiciones particulares en el que se tendrán en cuenta las normas legales y reglamentarias aplicables a las especificaciones técnicas propias de la obra de que se trate, así como las prescripciones que se habrán de cumplir en relación con las características, el empleo y conservación de máquinas, útiles, herramientas, sistemas y equipos preventivos.

El modelo español va más allá y prescribe la necesidad de elaborar los documentos gráficos necesarios, y define y evalúa económicamente los elementos y acciones de prevención y formación, en los apartados de Mediciones y Presupuesto.

Los aspectos de Seguridad, en el mantenimiento y las reparaciones del edificio, se han de contener en el Estudio.

En cuanto al Plan de Seguridad actualmente en vigor en España, puede decirse que cumple todos los requisitos establecidos en la Directiva de obras.

A nuestro juicio, la metodología y el contenido filosófico del decreto se encuentran dentro de lo establecido en la Directiva, siendo posible que en este caso la dirección de la convergencia sea del resto de los estados miembros hacia la situación española.

La ampliación del número de obras que precisarán de notificación, plan y expediente es aconsejable, pues es conocido que es en las obras pequeñas en las que el riesgo y el número de accidentes es mayor.

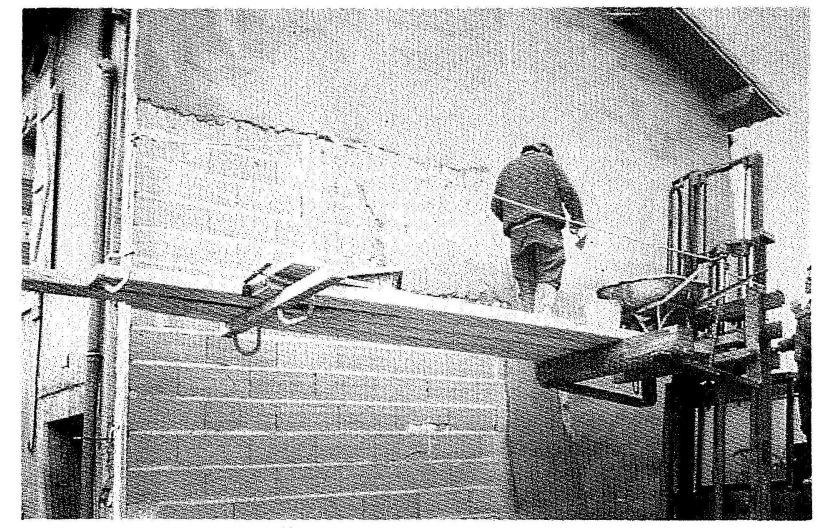

Fig. 1.-Los medios, equipos y máquinas han de ser montados, usados, mantenidos y desmontados según instrucciones del fabricante.

Conviene dimensionar el porcentaje de obras que actualmente están afectadas por el R.D. 555/86, aclarando que en la práctica el único parámetro que se aplica es el importe del presupuesto de la obra. Interesa también conocer la incidencia al costo destinado a seguridad, en relación con el presupuesto general de la obra.

Durante el año 1991, el Servicio de inspección del Colegio Oficial de Aparejadores y Arquitectos Técnicos de Madrid, procedió a analizar una muestra de 8.861 obras, de las que solamente el 10,32\%, o sea 915 , tenía un presupuesto superior a 100 millones de pesetas. La expresión gráfica de esa realidad se puede ver en la figura 1 .

Nos pareció interesante considerar qué importancia tenía el presupuesto total de ejecución material en el grupo de obras de importe superior a 100 millones, y el resultado fue el siguiente: De un total de $472.113 \mathrm{mi}-$ llones de pesetas de presupuesto total, de la muestra de obras analizadas, 367.950 millones correspondían al grupo de obras de más de 100 millones de pesetas de presupuesto de ejecución, es decir el 77,94 \% (Fig. 2).

Para analizar más minuciosamente la cuestión se tomó una muestra de 465 proyectos de prespuesto superior a 100 millones de pesetas y se procedió a dividir el grupo, según las diversas tipologías, acentuando la división en obras de viviendas unifamiliares adosadas y aisladas, por considerarlas de riesgo potencial más alto. La división se efectuó en los siguientes subgrupos:

1. Edificios de viviendas en altura (E. viviendas).

2. Viviendas unifamiliares aisladas (V. familiar). 


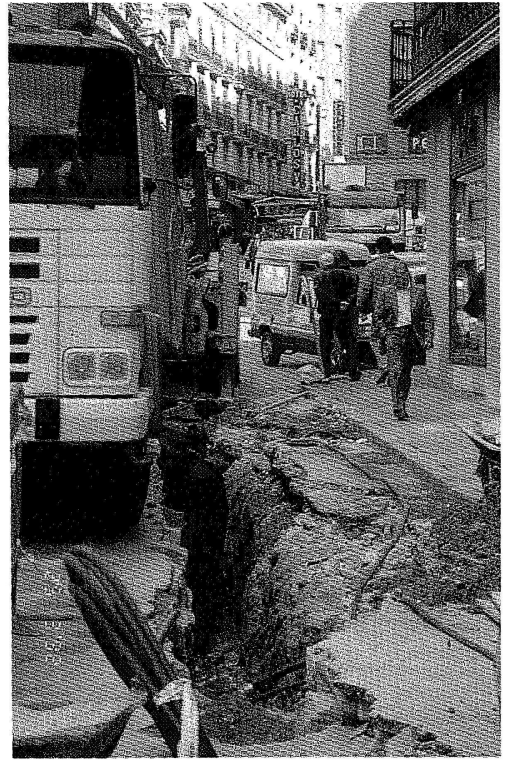

Fig. 2.-Las presiones en el entorno de las zanjas pueden causar graves daños, aunque la profundidad sea pequeña.

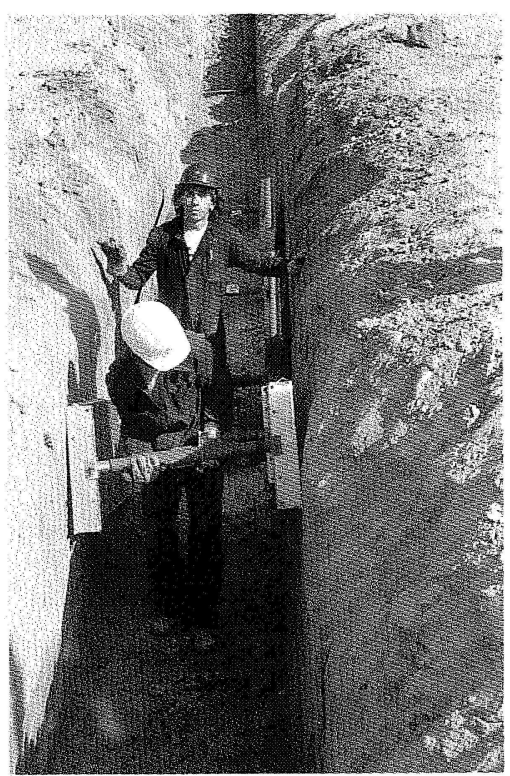

Fig. 3.-Las soluciones más simplesı son muchas veces las más eficaces.

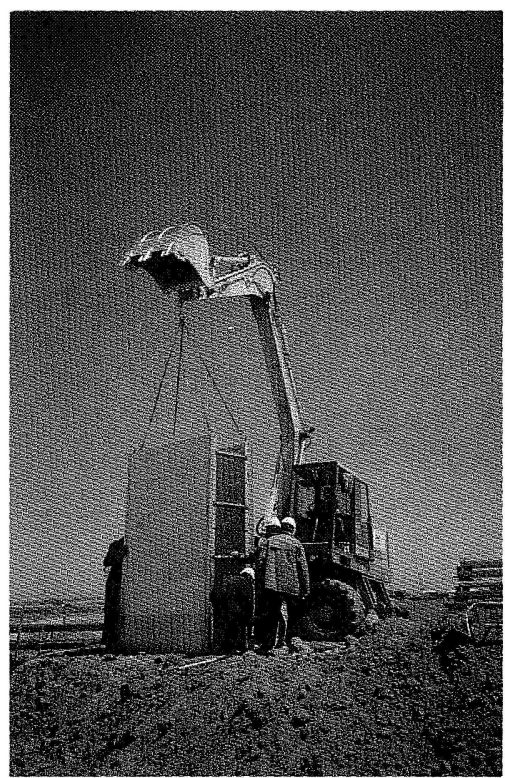

Fig. 4.-Cuando el potencial de riesgo sea alto deben utilizarse medios proporcionados.



Fig. 5.-En ocasiones, los riesgos son inducidos artificialmente.

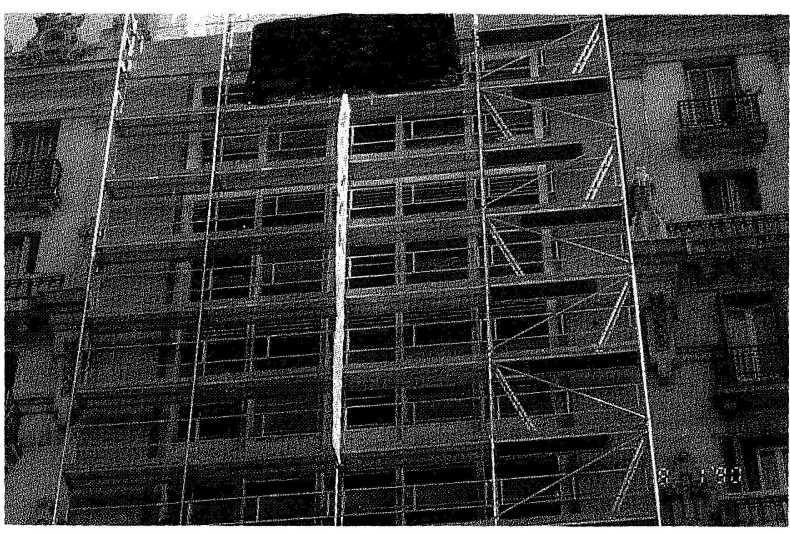

Fig. 6.-La seguridad debe estar presente también en el montaje de los andamios.
3. Viviendas unifamiliares adosadas (V. adosadas).

4. Edificios de oficinas (E. oficinas).

5. Naves industriales (Naves).

6. Aparcamientos (Aparcamientos).

7. Obras de urbanización (Urbanización).

8. Obras de reforma, rehabilitación (Reforma-rehabil.).

9. Otras tipologías (Otros).

Se evaluó el importe del presupuesto de ejecución material de cada subgrupo con el resultado de la figura 3.

A los efectos de proceder a comparar los importes de los presupuestos de obra, y los presupuestos contenidos en los estudios de seguridad, se determinaron los valores medios de presupuesto de obra por tipologías, según el resultado de la figura 4.

Es decir, que el valor medio del presupuesto de ejecución material de los proyectos de obras de más de 100 millones de pesetas es de 401,6 millones.

Las obras de edificios de oficinas tinen un importe medio de 773,7 millones, siendo los más elevados, y las obras de viviendas adosadas son las de menor importe, con un valor medio de presupuesto de ejecución material de 211,2 millones. 


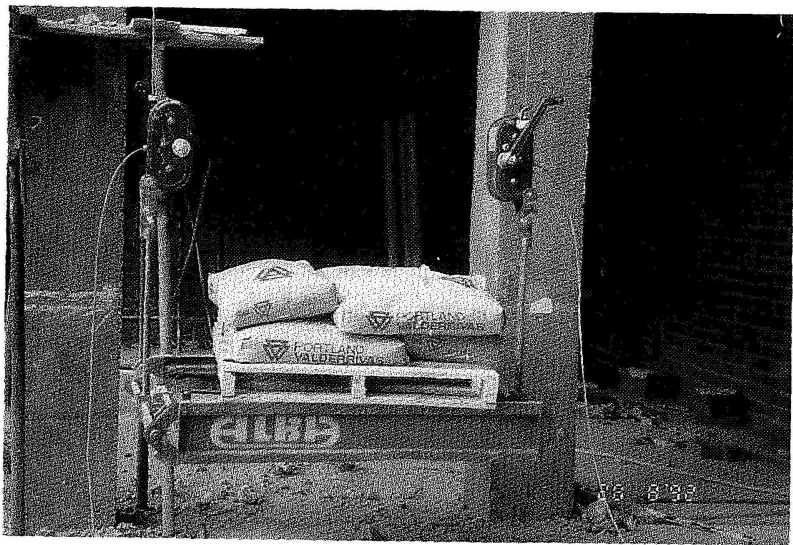

Fig. 7.-La legislación en vigor exige la prueba de carga en los andamios.

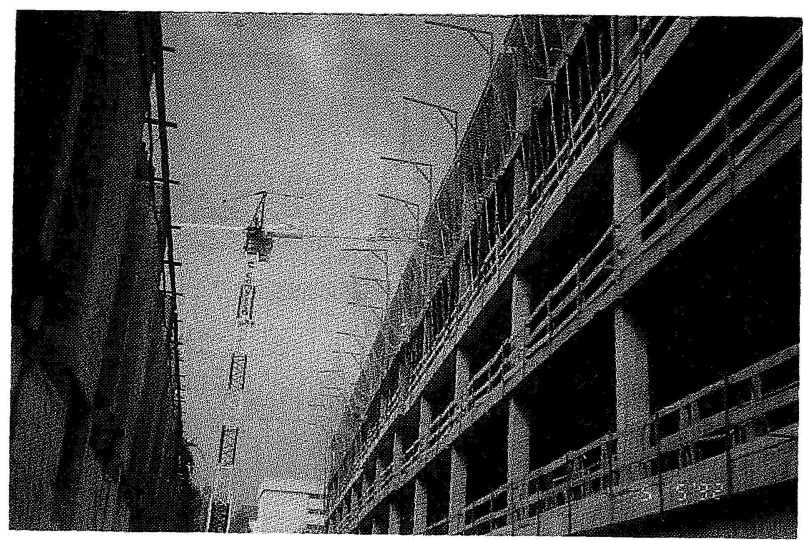

Fig. 8. - El mantenimiento sistemático de las protecciones colectivas hace descender el riesgo.



Fig. 9. - Las maniobras de elevación y el acopio de materiales concentran alto potencial de riesgo.

Conviene aclarar que en el subgrupo de viviendas unifamiliares aisladas, es frecuente que se redacten proyectos que contemplen varias viviendas aisladas, de ahí que el valor medio de este subgrupo de viviendas aisladas sea superior al de las viviendas adosadas.

La distribución porcentual de obras por tipologías se puede ver en la figura 5.

El análisis de los presupuestos de ejecución contenidos en los estudios de seguridad, por tipologías de obra, muestra que el valor medio es de 10,9 millones de pesetas, alcanzando el valor máximo de 20,5 millones para

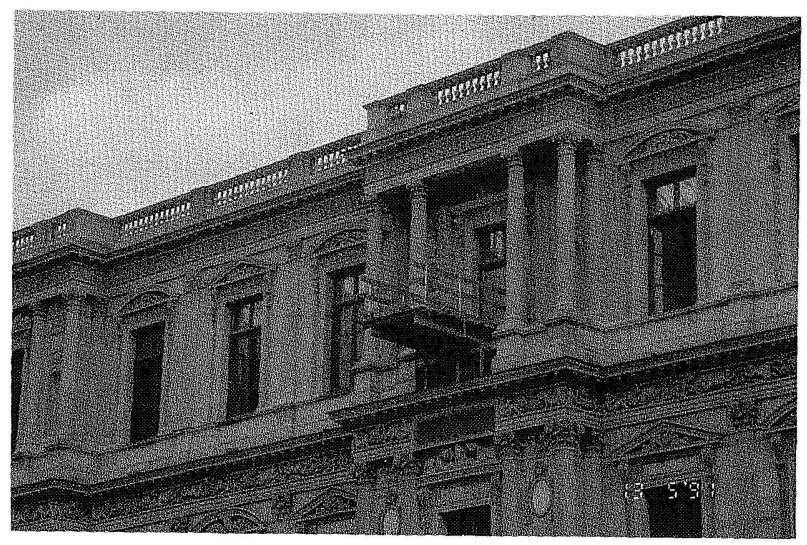

Fig. 10.-La plataforma de descarga hace más seguras las maniobras y mejora los rendimientos de los equipos de elevación.

el subgrupo de edificios de oficinas y el mínimo de 5,9 millones para el subgrupo de viviendas unifamiliares adosadas. Estos valores medios se reflejan en la figura 6 .

Era en este punto obligada la comparación entre los presupuestos de ejecución material de obra y los importes evaluados con destino a unidades de seguridad contenidos en los estudios, resultando que el valor medio de PEMS/PEMO es de 2,7 \%, siendo el porcentaje máximo el que corresponde a obras de reforma y rehabilitación, con un valor de 3,07\% y el mínimo $2,33 \%$ relativo a Obras de urbanización, siendo los restantes valores los que se señalan en la figura 7 . 


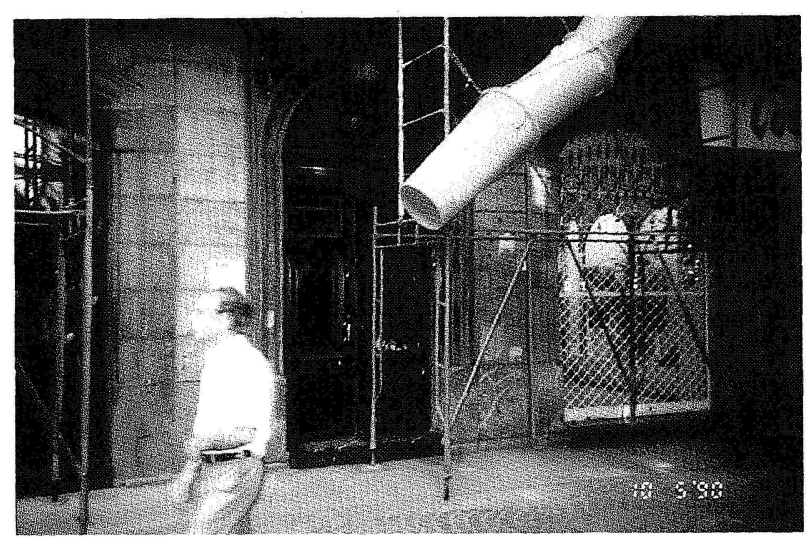

Fig. 11.-Las agresiones a los viandantes han de ser lo más leves posibles.

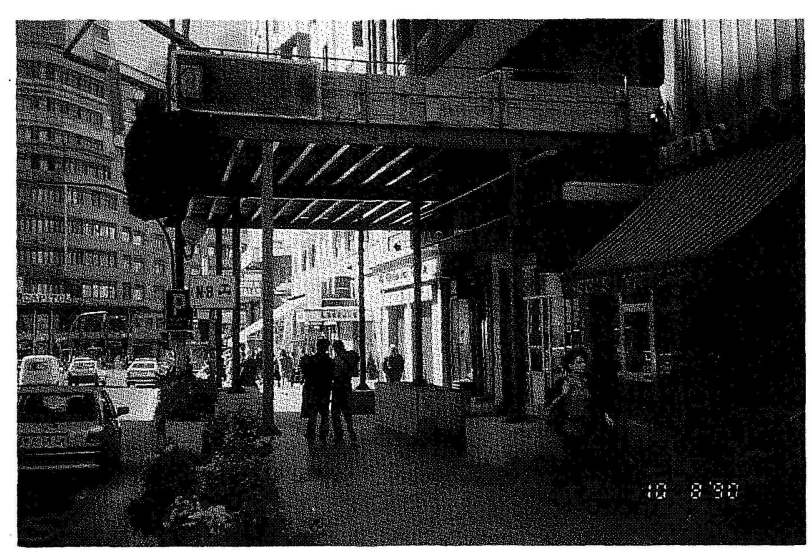

Fig. 12.-Las protecciones de la vía pública han de ser proporcionales al riesgo.

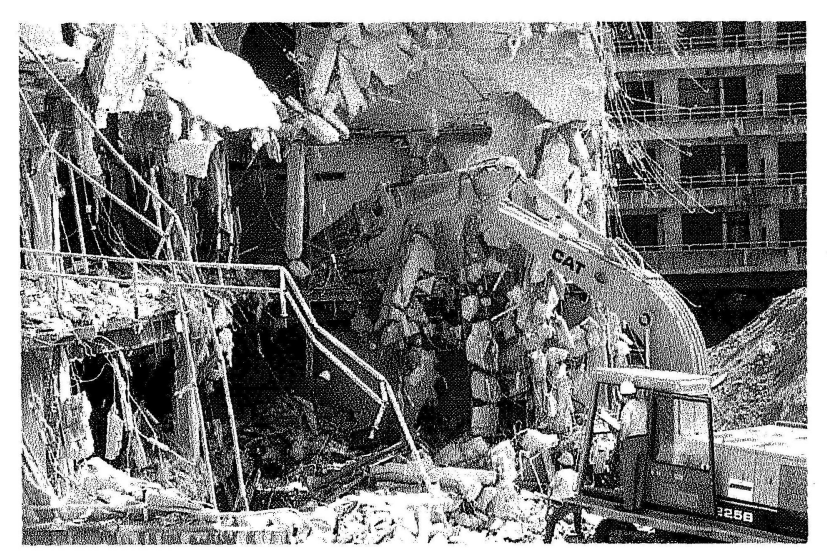

Fig. 13.-Algunas conductas son difícilmente explicables.
Otro aspecto que nos pareció interesante estudiar fue la distribución que se producía por tramos porcentuales de la comparación de presupuestos de obra y los presupuestos de Seguridad, obteniendo los siguientes resultados:

Obras cuya relación PEMO/PEMS:

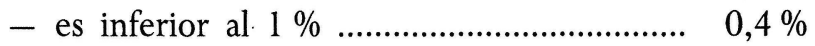

- está entre el $1 \%$ y $2 \%$.............................. 11,6\%

- está entre el $2 \%$ y $3 \%$................................40,9\%

- está entre el $3 \%$ y $4 \%$............................. 35, $1 \%$

= está entre el $4 \%$ y $5 \%$........................... $8,6 \%$

- es mayor que el $5 \%$.................................. 3,4\%

Ver figura 8.

Omitimos efectuar las correcciones resultantes del hecho probado de que el valor real de la ejecución de las obras es un $25-30 \%$ superior al que figura en los proyectos.

Conviene recordar, que la obligación de cumplimiento de las disposiciones vigentes en materia de seguridad y salud, es para todo tipo de obras, sean o no mayores de 100 millones de pesetas, pero no es menos cierto que concretar los aspectos preventivos, tanto en la fase de proyecto con el Estudio, como en la de ejecución con la elaboración del Plan y su control y seguimiento, sólo se realiza en las obras afectadas por el R.D. 555/86; por ello consideramos conveniente y acertada la nueva orientación que se contiene en la Directiva de Obras recién aprobada, por cuanto que extiende su aplicación a la práctica totalidad de las obras, subsistiendo la metodología, filosofía, instrumentos y agentes actualmente en funcionamiento, con las correcciones derivadas del volumen y características de los distintos tipos de obras.

El éxito o fracaso queda pendiente de las disposiciones en que se materialicen de la transposición de la Directiva y, sobre todo, del cumplimiento de lo preceptuado. 